\title{
The paramount importance of serological surveys of SARS-CoV-2 infection and immunity
}

\author{
Jaap Goudsmit ${ }^{1,2}$
}

Received: 9 April 2020 / Accepted: 11 April 2020 / Published online: 21 April 2020

(c) Springer Nature B.V. 2020

In this Commentary I will argue that repeated large-scale SARS-CoV-2 antibody testing of randomly-selected populations is a necessity during lifting of coronavirus pandemic restrictions. The current pandemic of COVID-19 caused by the coronavirus SARS-CoV-2 is being managed worldwide with different levels of social distancing and enhanced hygiene. The overall objective is to flatten the epidemic curve of symptomatic SARS-CoV-2 infections [1] below the level of maximum hospital capacity, in particular of the capacity of intensive care units.

This worldwide strategy of reducing social mixing has enormous impact on the economies of countries urging policymakers to contemplate how to open up sectors of the economy or the economy as a whole. The key question is: what data do we need to avoid a second wave of the pandemic larger and more extensive than what we have seen sofar? The general idea among epidemiologists and virologists is that a second wave that hits us late allows us to strengthen the infrastructure to track and trace contacts and contain the epidemic in such a way that the capacity of the health care system is more than sufficient to care for the severily ill. Most governments are wrestling with the principal choice between two epidemiological alternatives: primarily preventing infections versus primarily preventing the life-threatening consequences of infection. The first strategy focuses on the spreaders of infection and therefore on reducing the basic reproductive number or R0, the second on protecting the groups with the highest risk of morbidity and mortality.

Most lessons are learned from the epidemic in Wuhan, China. Studying 25,961 laboratory-confirmed COVID-19

Jaap Goudsmit

jgoudsmit@hsph.harvard.edu

1 Departments of Epidemiology and Immunology and Infectious Diseases, Harvard T.H.Chan School of Public Health, Boston, MA, USA

2 Human Vaccines Project, Boston, MA, USA cases with a median age of 57 years, Wang and coworkers [2] calculated that the $\mathrm{R} 0$ dropped from 3.86 before interventions to 0.32 post interventions in 18 days. The strict control measures involved control of the source of infection, cut off transmission routes and protection of vulnerable populations. Mathematical modeling by Prem et al. [3] indicated that the physical distancing measures were most effective if the return to work was staggered and initiated in early April, more than 2 months after the measures in Wuhan were fully implemented. The modeling also indicated that premature and abrupt lifting of interventions led to an early secondary wave of infections. However, the authors concluded that their analysis was limited by uncertainties around estimates of R0 and the duration of infectiousness. Gradual lifting of social distancing measures was initiated in Wuhan in early April.

The American Enterprise Institute (AEI) released a report on March 28, 2020 entitled "National Coronavirus Response, A road Map to Reopening" [4] that defined triggers to move from the full implementation of social distancing measures to normalization of people's life. All is targeted towards postponing the second wave and ameliorating the severity of cases requiring hospitalization and intensive care treatment. According to the AEI report the trigger to move from the full restrictive fase to the fase of lifting restrictions is "at least 14 days of sustained reduction in cases (the down slope of the epidemic curve), and hospitals are able to treat all patients without resorting to crisis standard of care, and the capacity to test for virus in the nose and throat of all people with COVID-19 symptoms as well as the ability to actively monitor active cases and contacts. At that point the majority of schools, universities and businesses can gradually reopen, the report suggests. Contact tracing from then on is key. Working from home and enhanced hygiene measures should stay in place including limiting gatherings of more than 50 people for the whole population in this fase and all physical distancing measures should stay in place in full for the most vulnerable, the people over 60-years of age 
and all with co-morbidities and underlying compromised immune systems or lung function. Lastly serological testing should be in place to identify who has recovered from infection and is potentially protected from re-infection. The report of the American Enterprise Institute suggests that the final and last restrictive measures can only be lifted if either therapies for the most vulnerable or a vaccine is available. This defines the timeline somewhat: the restrictive fase may last 2-3 months, the loosening up fase lasts till we have therapies or a vaccine to protect the highest risk groups against disease. Because of the variable length of the second fase of gradual lifting of the precautionary measures, this is the fase we have to focus on now while therapies and vaccines are in expedited development in a parallel track.

During this second fase the most important information we need is: who are still susceptible to infection, who are acutely infected and infectious and who are recovered and potentially immune to re-infection [5].

To get this information large-scale serological surveys are of paramount importance [5]. This is particularly true if a major proportion of SARS-CoV-2 infected individuals do not experience any symptoms as is currently assumed. Large-scale randomly selected studies would be able to identify such individuals while the current way of testing for acute symptomatic infections does not. This would also solve the problem of overfitting the trajectories of the epidemic curve based on the number of symptomatic infections instead of on the total number of infections including the asymptomatic ones [6]. The current prediction is that there are approx. 10 times more people infected than the number of symptomatic cases confirmed by demonstrating virus in the upper respiratory tract [7]. Leveraging serological testing might be the most effective way to complement or even replace social distancing by a strategy called "Shield Immunity", focusing on recovered individuals and assuming that recovered individuals are immune to re-infection and therefore will be no longer a source of new infections [8]. The strategy of "Shield Immunity" is based on amplifying the proportion of interactions with recovered individuals relative to those with individuals of unknown status, in other words let people who recovered from a proven SARS-CoV-2 infection based on a blood test interact freely. The complicating factor for this strategy to be effective is that it is hard to identify everybody who has protective antibodies in their blood because this is time-dependent, requiring repeat testing and coverage-dependent,- how many people are immune at start of lifting restrictions and how many during the gradual abandoning of the restrictions-.

Immunity against re-infection following a proven SARS$\mathrm{CoC}-2$ infection has been equated to the presence of antibodies to the crown protein or spike and more specifically to the receptor binding domain (RBD) able to neutralize the virus. It has been shown that the plasma level of such neutralizing antibodies binding to the spike plateaus early in infection and are higher in elderly than in young patients that recovered from a symptomatic SARS-CoV-2 infection and $30 \%$ of the people who recovered did so without detectable neutralizing antibodies [9]. This absence of neutralizing capacity while binding antibodies to the virus are seen in all infected and recovered individuals could mean that the neutralizing antibody test is not sensitive enough or that a function other than virus neutralizaton of spike or virus binding antibodies confers protection. This phenomenon has been seen with the monoclonal antibody CR9114 binding to the influenza hemagglutinin stem and protecting mice against challenge with influenza B strains through antibody-dependent cellular cytotoxity (ADCC) in the complete absence of virus neutralization [10].

In conclusion, during the period of relaxation of pandemic precautionary measures awaiting effective treatment of the severe cases of COVID-19 and/or a vaccine conferring protection against SARS-CoV-2 infection and disease, large- scale testing for antibodies to SARS-CoV-2 is essential. The serological testing of randomly selected populations nationwide should be repeated at regular intervals to identify new infections by seroconversion, by IgM positivity or by four-fold IgG rise in titers [11]. Vulnerability for infection is reflected by the absence of any antibody and immunity by a stable IgG titer in the absence of IgM. Stratifying risk of infection and level of protection associated with residence, -urban vs rural-, socio-economic group, disease and health predictors, and age and sex, allows policymakers to make informed decisions on the strategy of lifting pandemic restrictions.

\section{References}

1. Dalton CB, Corbett SJ, Katelaris AL. Pre-emptive low cost social distancing and enhanced hygiene implemented before local COVID-19 transmission could decrease the number and severity of cases, MedRxiv, 2020.

2. Wang C, et al. Evolving Epidemiology and Impact of Non-pharmaceutical Interventions on the Outbreak of Coronavirus Disease 2019 in Wuhan, China, MedRxiv, 2020.

3. Prem K, et al. The effect of control strategies to reduce social mixing on outcomes of the COVID-19 epidemic in Wuhan, China: a Modelling Study, Lancet Public Health, March 25, 2020.

4. American Enterprise Institute, National Coronavirus Response, A Road Map to Reopening, March 28, 2020.

5. Lourenco J, et al. Fundamental principles of epidemic spread highlight the immediate need for large-scale serological surveys to assess the stage of the SARS-CoV-2 epidemic, MedRxiv, 2020.

6. Lancastle $\mathrm{N}$. Is the impact of social distancing on coronavirus growth rates effective across different settings? A non-parametric and local regression approach tot test and compare the growth rate, MedRxiv, 2020. 
7. Pedersen MG, Meneghini M. A simple method to quantify country-specific effects of COVID-19 containment measures, MedRxiv, 2020.

8. Weitz J, et al. Intervention Serology and Interaction Substitution: Modeling the Role of 'Shield Immunity' in Reducing COVID-19 Epidemic Spread, MedRxiv, 2020.

9. Wu F, et al. Neutralizing antibody responses to SARS-CoV-2 in a COVID-19 recovered patient cohort and their implications, MedRxiv, 2020.

10. Cox F. HA antibody-mediated FcgammaRIIIa activity is both dependent on FcR engagement and interactions between HA and sialic acid. Front Immunol. 2016;7:399.
11. Long Q, et al. Antibody responses to SARS-CoV-2 in COVID-19 patients: the perspective application of serological tests in clinical practice, MedRxiv, 2020.

Publisher's Note Springer Nature remains neutral with regard to jurisdictional claims in published maps and institutional affiliations. 